\title{
BCG Tokyo-172 Strain Solution
}

National Cancer Institute

\section{Source}

National Cancer Institute. BCG Tokyo-172 Strain Solution. NCI Thesaurus. Code C126694.

A solution containing an attenuated, live culture preparation of the bacillus CalmetteGuerin (BCG) strain of Mycobacterium bovis obtained from the Pasteur Institute in 1924, with potential immunostimulating and antineoplastic activities. Although the precise mechanism of action is unknown, upon intravesical instillation through a catheter, the attenuated, live BCG bacteria in the BCG Tokyo-172 strain solution come into direct contact with the bladder wall and elicits a local, multifaceted immune response against the BCG antigens, which kills the bladder cancer cells. Previous vaccination with a systemic BCG vaccine may enhance the immune system's response against the BCG antigens. 